\title{
AN ARC-LENGTH SOLVER WITH DISSIPATION PATH-FOLLOWING FOR COMPLEX ANALYSIS OF BRITTLE FAILURE AND STABILITY OF COMPOSITE STRUCTURES.
}

\author{
Elias I. Börjesson ${ }^{1 *}$, Martin Fagerström ${ }^{1}$ and Joris J.C. Remmers ${ }^{2}$ \\ ${ }^{1}$ Dept. Industrial and Materials Science, Chalmers University of Technology, Gothenburg, Sweden \\ ${ }^{2}$ Dept. Mechanical Engineering, Eindhoven University of Technology, Eindhoven, The Netherlands \\ * elias.borjesson@chalmers.se
}

The failure of laminated, fibre-reinforced structures generally involves the interaction of several failure mechanisms, many of them with a brittle behaviour including rapid softening. The damage models used to simulate these failure mechanisms often creates numerical difficulties for the solution method. For example, standard Newton solvers are generally unsuitable for cases where rapid failure occurs in parts of the structure. A remedy often resorted to in the literature, is to include dynamical effects via implicit or explicit time integration. However, an alternative approach, not penalised by requiring very small time steps, mass-scaling and/or introducing unwanted inertia or other dynamical effects, is to adopt an arc-length based quasi-static solver.

Much research has been made in the field of arc-length solvers, and there is now a range of available methods. Traditional approaches base the equilibrium path-following constraint on the norm of the incremental displacement vector (i.e. an arc-parameter). These methods perform well for problems exhibiting geometrical non-linearities, but have difficulties converging when material instabilities are involved. In order to remedy this, a path-following constraint based on the energy release rate for geometrically linear continuum damage was proposed in [1]. The method calculates the energy release rate using global quantities (such as internal force and displacement vectors), and was shown to give robust and stable convergence for problems with advanced stages of damage. The methods was later extended in [2] to include geometrically non-linearity, with plasticity and damage computations.

In the current contribution, we reformulate the dissipation based path-following equation proposed in [1], from being calculated with global quantities, to instead being determined from the material dissipation. This has the added benefit of unifying the path-following constraint for all types of dissipative problems, and no adjustment of the solver to specific problem cases (linear/non-linear, plasticity/damage etc.) is needed. The stability and robustness of the solver is showcased in numerical examples with composite structures that experience large non-linear damage behaviour and damage driven buckling.

\section{References}

[1] Gutiérrez, M.A. (2004). Energy release control for numerical simulations of failure in quasi-brittle solids. Communications in Numerical Methods in Engineering, 20, 19-29.

[2] Verhoosel, C. V., Remmers, J.J.C., Gutiérrez, M. A. (2009). A dissipation-based arc-length method for robust simulation of brittle and ductile failure. International Journal for $\mathrm{Nu}$ merical Methods in Engineering, 77, 1290-1321 